\title{
17
}

\section{Developing Best Estimates for CSO Control Volumes to Meet NPDES Requirements}

\author{
Robin J. Lee, Tai Ovbeibo, Joseph Pang, Mel Schaefer, Bruce Bark- \\ er, Ben Marré and Steve Merrill
}

The City of Seattle owns and operates a combined sewer system (CSS) that overflows during heavy rain events into surrounding water bodies, potentially impacting their quality and uses. Hydraulic and hydrologic models of the city's uncontrolled combined sewer overflow (CSO) basins were developed to identify projects and programs that will limit untreated overflows at each CSO outfall to an average of no more than one annually, a performance standard established in the City's CSO national pollutant discharge elimination system (NPDES) permit.

After manual calibration, each model underwent an automated calibration process, the automated calibration and uncertainty analysis for storm water management model (ACU-SWMM). ACU-SWMM is a software package created by MGS Engineering Consultants, Inc. (MGS) for use with the U.S. Environmental Protection Agency (USEPA) Storm Water Management Model (SWMM5). It was designed primarily for use with CSSs where uncertainties from multiple sources can make model calibration difficult and severely impact the reliability of sewer flow predictions.

This chapter also describes the process established to identify the overflow control levels using a continuous long term simulation (LTS), taking into account the effect of the several uncertainties due to precipitation time series, collected flow data, climate changes, and model hydrology and hydraulics uncertainties.

Lee, R., T. Ovbiebo, J. Pang, M. Schaefer, B. Barker, B. Marre and S. Merrill. 2012. "Developing Best Estimates for CSO Control Volumes to Meet NPDES Requirements." Journal of Water Management Modeling R245-17. doi: 10.14796/JWMM.R245-17.

(C) CHI 2012 www.chijournal.org ISSN: 2292-6062 (Formerly in On Modeling Urban Water Systems. ISBN: 978-0-9808853-7-8) 


\subsection{Model Development}

This section describes the process of model development, from collection of data through model building and hydraulic calibration.

\subsubsection{Background}

Hydraulic and hydrologic models of Seattle's uncontrolled CSO basins were developed to identify projects and programs that will limit untreated overflows at each CSO outfall to an average of no more than one per year, a performance standard established in the city's CSO NPDES permit. This work was done as part of the city's long term control plan (LTCP), which will define the CSO reduction projects. The models were constructed using the SWMM5 platform to enable the city to investigate retrofit opportunities, green stormwater infrastructure (GSI) projects and traditional infrastructure to reduce the city's CSO volume.

\subsubsection{Flow Monitoring Data Collection}

Flow monitoring data were collected from October 2008 through May 2010, from 250 temporary monitoring locations operated by Stantec and an additional 100 permanent CSO monitoring sites operated by ADS Environmental Services (flow monitoring contractor). This information was used to develop dry weather flow (DWF) estimates, calibrate hydraulic structures (e.g., HydroBrakes, weirs, pump stations and storage pipes), and calibrate and validate wet weather response.

Throughout the flow monitoring period, the data were used to compute flow balances to ensure that individual meters were recording data properly. The city invested \$8 million (of which \$1 million was invested in equipment) in this flow monitoring program to ensure accurate data collection. The program was successful because at the outset, a clear statement of goals and objectives was made for the project, and data quality usability standards were set to ensure high quality data. In addition, the overall success resulted from the recognition that the first year of the program was not going to provide adequate data due to an insufficient number of wet weather events; the program was then modified to include a second wet weather season. This data were vital to the automated calibration and manual hydraulic calibration processes.

\subsubsection{Model Building and Hydraulic Calibration}

The city's CSS is mapped in the geographic information system (GIS). Pipes and nodes were imported into the SWMM5 models from GIS data. Subcatch- 
ments were also developed using GIS data for buildings, parcels, pavement, sidewalks, and rights of way. Individual hydraulic structures were entered manually into the models. The most unique structures in the Seattle system are HydroBrakes. HydroBrakes are a self-activating vortex flow control device. Each HydroBrake structure has a manufacturer stage-discharge curve that shows flow as a function of hydraulic head. Flow monitoring was set up around these structures to collect upstream depth measurements and downstream velocity and depth measurements. In some cases, the collected data showed a different curve than what was predicted by the manufacturer. Figure 17.1 below shows an example of when the data did not match what the manufacturer's curve suggested. HydroBrakes were modeled as an outlet with a rating curve from observed data.

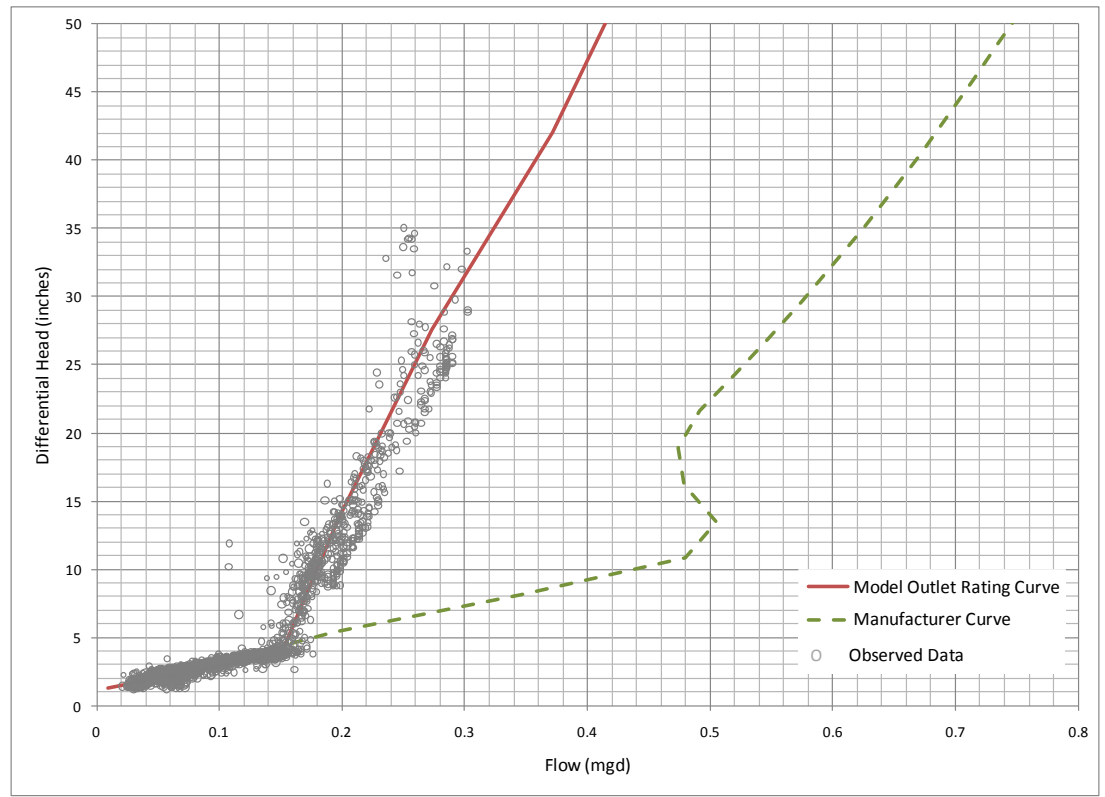

Figure 17.1 Single HydroBrake curve

In some cases, it was observed that the curve shifted to the right (higher flow) when the downstream side of the HydroBrake was submerged. When this case was observed, an orifice or multiple outlets with a control rule was used to model the different curves. Figure 17.2 below shows two different curves.

Some of the initial orifice and outlet curves needed to be modified after automated calibration. 


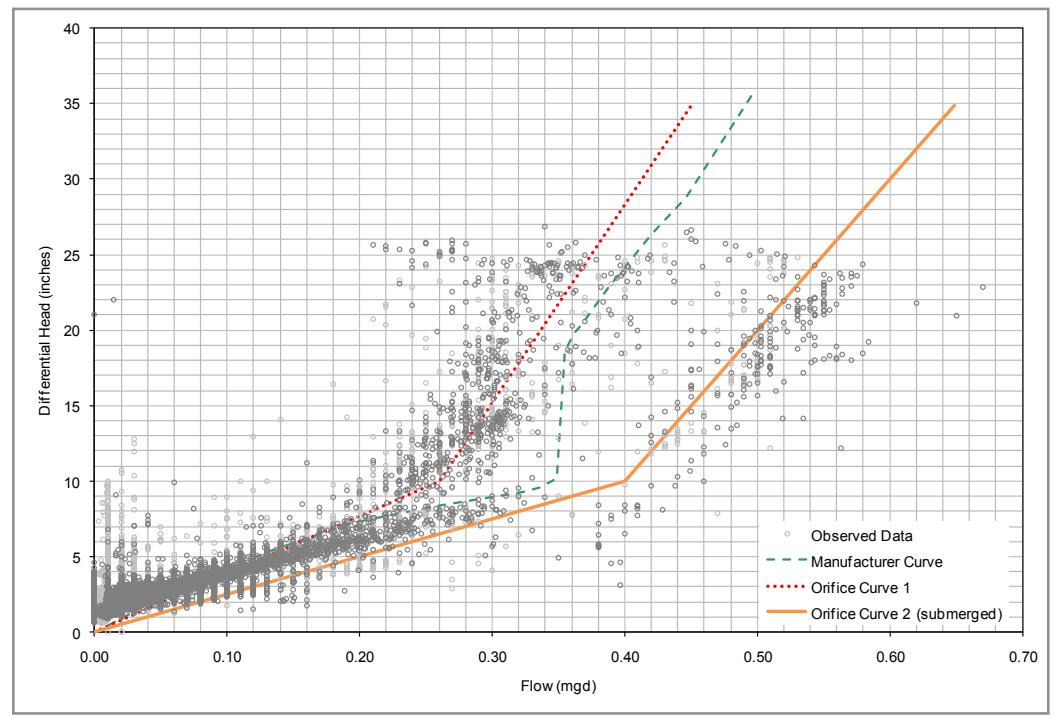

Figure 17.2 Dual HydroBrake curve.

\subsection{Automated Calibration}

Once the models were built for each of the uncontrolled CSO basins, they underwent an automated calibration process called ACU-SWMM, a software package created by MGS for use with SWMM5. It was designed primarily for use with CSSs where uncertainties from multiple sources can make model calibration difficult and severely influence the reliability of sewer flow predictions.

\subsubsection{ACU-SWMM Functions and Modules}

ACU-SWMM has two primary functions. First, it is intended for automated calibration of SWMM5 models of urbanized basins. Second, it computes a control volume (CV) and uncertainty bounds for the CSO volume with a onceper-year frequency of occurrence. A CV is the overflow volume exceeded once per year on average. This second function is discussed in Section 17.3.

ACU-SWMM has four major modules; two modules are for automated calibration and two modules are for conducting an uncertainty analysis for estimating the CSO CV. Section 17.3 describes these latter two modules. Detailed descriptions of the computational procedures for automated calibration and uncertainty analysis are not presented here. Chapters 5 and 6 of the CSO Tech- 
nical Guidance Manual (MGS, 2010) provide a detailed description of the computational procedures.

Module 1 of the automated calibration process includes all of the tasks for project description, file management, data management, identification of flow and depth meters used for calibration, identification of model parameters used for calibration, setting of sampling ranges of parameter values, and conducting numerous simulations by executing the SWMM5 basin model in a batch mode.

Module 2 (see Figure 17.3) of the automated calibration process includes computation of the numerical goodness of fit (GOF) measures for comparison of recorded and computer-simulated hydrographs.

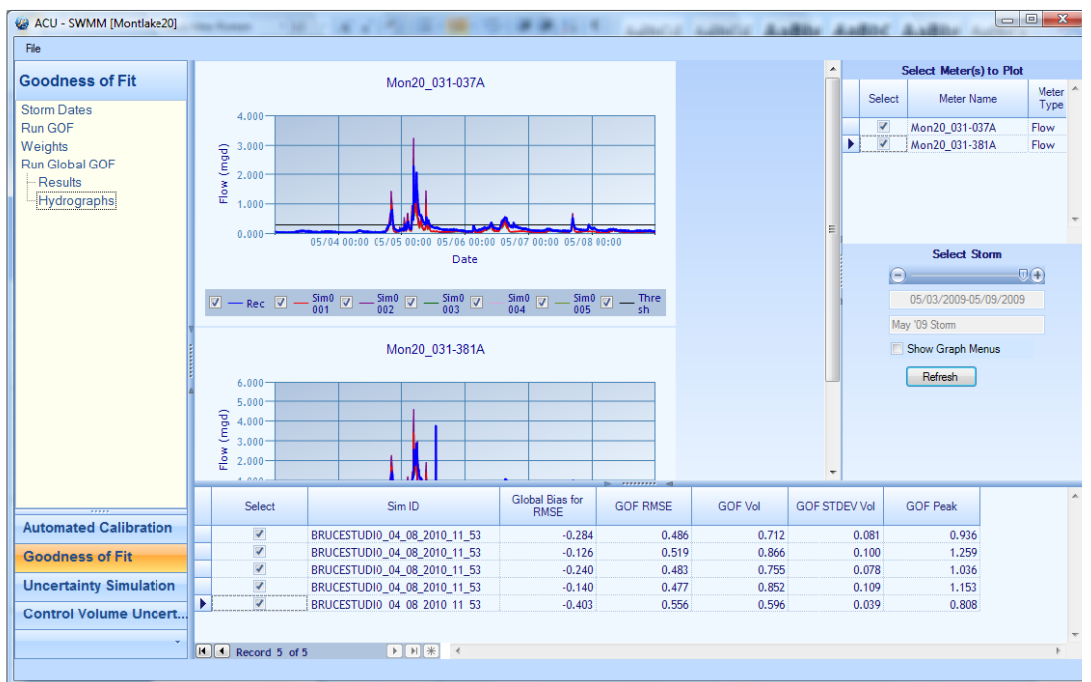

Figure 17.3 User interface for ACU-SWMM module 2.

This module allows the user to perform the following tasks:

1. Weight the relative importance of specific flow and depth meters and specific storm events for use in computing global GOF measures, which provide the best fit of computer simulated hydrographs to the hydrographs that have been recorded at various sites throughout the model;

2. Compute global GOF measures reflecting the combined importance of the various meters and storms (e.g., the user defined weights);

3. Sort SWMM5 model parameter sets in order of global GOF measures; and 
4. View graphical representations of recorded and simulated hydrographs for selection of the best fit model parameter set.

\subsubsection{Automated Calibration Sampled Parameters and Ranges}

The automated calibration procedure utilizes a Monte Carlo sampling approach similar to that used in the generalized likelihood uncertainty estimation (GLUE) approach initially developed by Beven and Binley (1992). Additional information on this approach is described in the CSO Technical Guidance Manual (MGS, 2010). The Parameter Sample component is used to identify the probability distribution, and minimum and maximum values of the model parameters that will be used in automated calibration. The significant steps in the ACUSWMM process are outlined below:

1. Seven SWMM5 hydrologic model parameters were identified to be sampled for the models. Table 17.1 summarizes the parameters and the user defined sampling ranges for one of the basin models. These ranges were modified after the initial calibration runs as the range of parameters was tightened;

2. Maximum and minimum values for each parameter were specified to limit sampling to a realistic range of values;

3. ACU-SWMM used a Monte Carlo sampling technique to select from several hundred to several thousand sets of parameter combinations from within the user defined ranges. SWMM5 was then called to run for the duration of the observed flow data with each of the selected parameter combinations. This was often an iterative process where the GOF results from one round of sampling were used to narrow the parameter sampling ranges for the next round of sampling. The simulated output was compared to the observed flow measured for each defined calibration event (storm event);

4. Weighting factors were applied to specific calibration events;

5. Percent of recorded volume (above a user defined threshold), percent of recorded peak, and standard deviation of percent of recorded volume were used to compute the GOF between the simulated and observed data for flow and depth above a given threshold value for the duration of the calibration rainfall events; and

6. The parameter sets were ranked by GOF to identify the candidate best fit parameter sets for consideration in the final calibration model. 
Table 17.1 Parameters and initial sampling ranges for ACUSWMM.

\begin{tabular}{|c|c|}
\hline Hydrologic parameter & Sampling range \\
\hline \% Impervious (building subcatchments only) & $\pm 20 \%$ of GIS average \\
\hline Green-Ampt conductivity (in./hr) & $0.04-0.43$ \\
\hline Groundwater flow coefficient, A1 & $0-0.3$ \\
\hline Groundwater flow exponent, B1 & $1.0-3.5$ \\
\hline $\begin{array}{l}\text { Aquifer water table elevation, distance below subcatchment } \\
\text { threshold (ft) }\end{array}$ & $\pm 0-0.01$ model value \\
\hline $\begin{array}{l}\text { Aquifer bottom elevation, distance below initial water table } \\
\text { elevation (ft) }\end{array}$ & $\pm 0.3-0.8$ model value \\
\hline Aquifer lower groundwater loss rate (in./day) & $0.3-0.7$ \\
\hline
\end{tabular}

\subsubsection{Automated Calibration Meter Thresholds and Calibration Groups}

Each model was calibrated in a downstream direction. The results of the upstream calibrations were used as inflows to the lower meters. The ACUSWMM process focused on finding and locking in parameters for the upper calibration groups before focusing on the lower calibration groups. The calibration storms and threshold for a given flow meter are shown below in Figure 17.4.

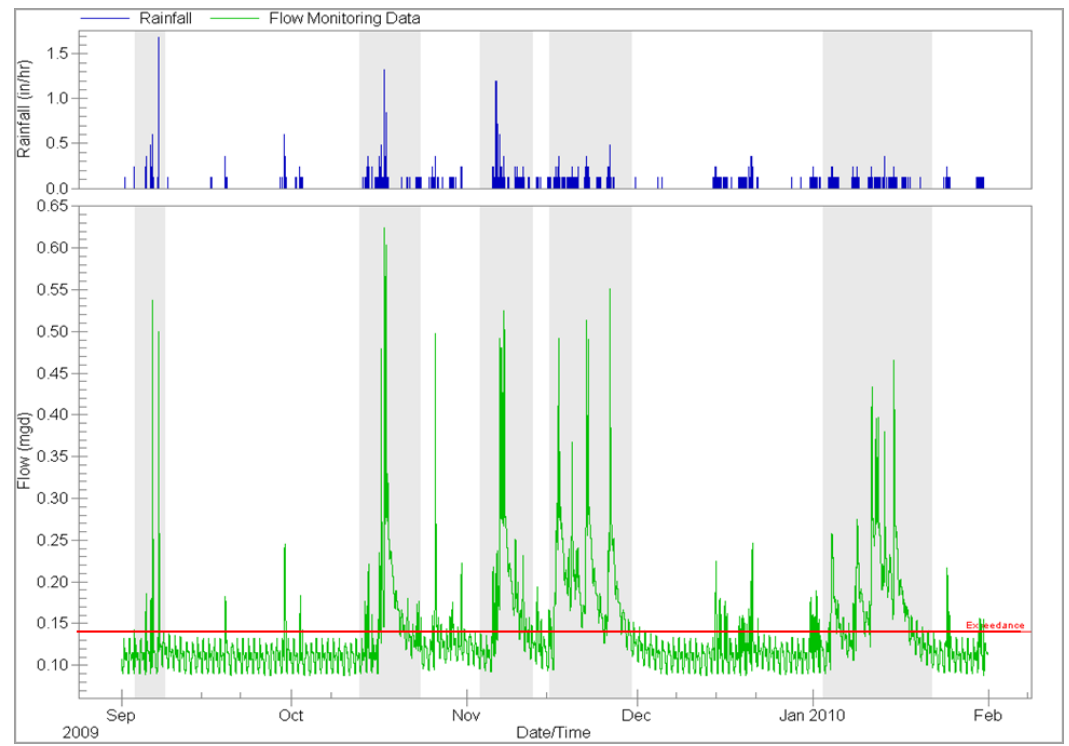

Figure 17.4 Calibration events (highlighted in grey) and threshold (shown in red). 
The model simulation results were compared to meter data for the portion of the output hydrograph that was above user specified threshold values when computing the ACU-SWMM GOF statistics. Prioritizing the calibration of storm flow results in a better matching of the hydrologic response during the events that are most likely to generate CSO events. To establish the thresholds for the GOF for each model, the meter data were reviewed to evaluate a flow value above the peak DWF, but below the wet weather component for each of the potential calibration storms. This process removed the noisy and unimportant DWF data in the GOF calculations.

\subsubsection{Numerical Goodness of Fit Measures}

The advantage of the ACU-SWMM automated calibration process is its ability to examine fully the range of parameter combinations within the range of plausible parameter values on model-generated sewer flow hydrographs using numerical GOF measures over the entire flow monitoring period. This approach is important because uncertainties in measurement of representative precipitation, measurement of sewer flows, and imperfections in model structure cloud the search for the best fit model parameter set. Finding the best fit parameter set by visually comparing simulated and recorded sewer flow hydrographs is not feasible given the large number of potential hydrographs. To simplify the identification of a best fit parameter set, a series of GOF measures is used to describe the overall comparison between a given set of computer simulated and recorded sewer flow hydrographs. Five separate GOF statistics are generated using the ACU-SWMM post processing tool:

standardized root mean square error (RMSE);

standardized bias RMSE;

percent of recorded volume;

standard deviation of percent of recorded volume; and

percent of recorded peak.

The GOF measures for sewer flow volume, peak discharge, and standard deviation of sewer flow volume were used in identifying the best fit model parameter set. The GOF measures for RMSE were not used in automated calibration because timing discrepancies of the time stamps for the precipitation gauge and sewer flow meters often rendered the RMSE measures unreliable.

Two types of GOF measures are computed. Event GOF statistics are computed for each meter-storm combination that represents comparisons of individual simulated and recorded hydrographs. Global GOF measures, which are computed using the event GOF measures, represent the overall GOF for the collection of meters and storm events. Global GOF measures are computed by applying user specified weights to the event GOF measures (see Equation 17.1). 
The global GOF statistics are used to screen many potential parameter sets and to help identify the best fit parameter set. The methods used to compute these statistics, as well as a more indepth discussion of GOF theory, can be found in the CSO Technical Guidance Manual (MGS, 2010).

$$
G O F_{\text {global }}=\sum_{m=1}^{M M}\left(\text { GOF event }_{m} * \text { Event }_{\text {Weight }}{ }_{m}\right)
$$

where:

$$
\begin{aligned}
m \text { to } M M= & \text { storm sewer flow events, } \\
G O F_{\text {event }}= & \text { goodness of fit calculated for each storm sewer flow } \\
& \text { event, and }
\end{aligned}
$$

EventWeight $_{m}=$ user defined weight applied to $m$ storm event.

Event weights, discussed further below, are used to give greater emphasis to events that caused overflows, events that were generally larger in rainfall volume, or events that contained significant features deemed important to the overall calibration.

\subsubsection{Automated Calibration Event and Meter Weighting Factors}

After completing a batch of simulations (typically 500), the calibration groups were post-processed to calculate a GOF statistic for each meter, parameter set and storm combination. Weighting factors that were assigned to the storm events and meters were incorporated into calculating a global GOF for each parameter set, which then served as a query tool to identify candidate best fit modeling parameter sets.

Initially, the team focused on evenly weighting the events and meters in the calibration groups to identify parameter sets that resulted in sufficient correlation to meter flow and depth measurement over a majority of storms. Using this method, the overall fit was often unacceptable. This situation highlighted the difficulties in identifying those combinations of meters and storm events for which there was confidence in the quality of the flow and storm data.

Prior calibration work on other basins within Seattle had shown that a suitable model parameter set could be found when both high quality flow data and precipitation representative of the flow monitoring subcatchment were apparent. Therefore, the inability to locate any parameter sets from approximately 500 simulations raised the possibility of problems with the flow data or representative precipitation, or a flaw in the model structure. Each of these possibilities was examined, revealing in some cases flaws in the model structure that were found and addressed. In other cases, the quality of the flow data or representativeness of the precipitation data was lacking and the meter or storm event was given lower weighting or was removed from consideration. 
With these issues in mind, the team concentrated on examining events where the model predictions and recorded data had a greater visual consistency and not on storms that did not compare well to the recorded data for all meters.

Weights were then applied to the combination of meters and storm events that reflected the modeling team's judgment of the quality of the flow and rainfall data and the importance of the storm event. In general, greater weight was given to larger sewer flow events, particularly where sewer overflows occurred. These weightings led to identifying parameter sets that were reasonably capable of replicating recorded sewer flows for the varying storm events. Large groundwater events occurred during late November 2009 and January 2010. These events were used to help refine the groundwater parameter calibration. Using this procedure led to a single set of parameters capable of accurate simulation of the system across a wide range of antecedent conditions and storm magnitudes. Thus the selected parameters are considered the most appropriate for long term continuous simulation to define compliance requirements.

\subsubsection{Best Fit Model Parameter Sets}

The best fit model parameter set for each subcatchment model was selected using a two step approach. First, candidate parameter sets were identified using global GOF measures for sewer flow volume, peak discharge, and standard deviation of volume. Next, a visual comparison was made of recorded hydrographs and simulated hydrographs produced by candidate parameter sets. Figure 17.5 shows an example model calibration hydrograph.

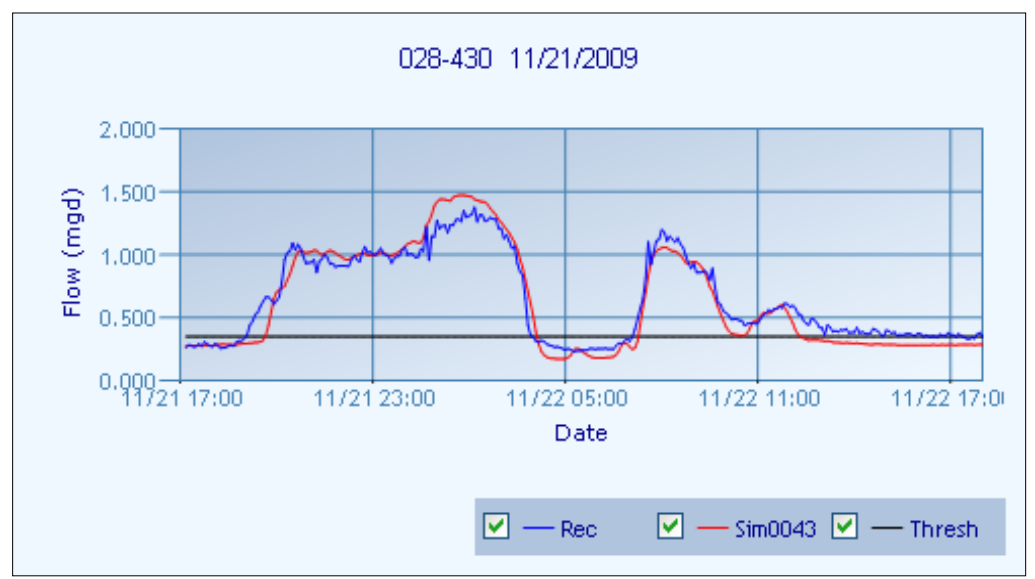

Figure 17.5 Example model calibration hydrograph (blue $=$ observed data; red $=$ simulated; black $=$ threshold for observed data). 
Particular attention was given to matching hydrograph shape, peak discharge, and the recession limb of the hydrograph. In particular, the slope and shape of the recession limb were useful in identifying groundwater parameters for groundwater inflow that was evident in the later portion of November 2009 and early January 2010. An important goal of the calibration process was to identify a best fit parameter set that yielded sewer flow predictions with low bias and low standard deviation for volume. A subcatchment model with low bias would have global GOF measures for volume and peak discharge near a value of $100 \%$. Good replication of recorded sewer flow hydrographs for the collection of storm events and meters would have a low global GOF for the standard deviation of volume. The global measure for standard deviation for volume was used later in the uncertainty analysis for characterizing the magnitude of model uncertainty, which is further discussed in Section 17.3.1.

\subsection{Long Term Simulation}

The basic approach to assess sewer system performance is to conduct LTSs using the calibrated basin model and the historical $32 \mathrm{y}$ precipitation time series from the nearby precipitation gauge. A CSO CV with a frequency of $1 / \mathrm{y}$ is determined by choosing the 32nd largest overflow volume from the $32 \mathrm{y}$ time series of computer simulated overflows. To account for uncertainty, eleven separate LTSs (chosen as a compromise between the desire for many runs to better define uncertainty and the time and resource intensity of the simulations) are conducted where the historical 32 y precipitation time series (1978-2009) is rescaled in a manner to reflect the magnitude of uncertainties estimated from several sources. This approach yields eleven plausible futures with regard to the manner in which uncertainties can affect the magnitude of sewer flows and overflow volumes. These eleven plausible futures allow determination of the best estimate CSO CV and uncertainty bounds. The ACU-SWMM software package was used to conduct the LTSs and compute CSO CVs and uncertainty bounds for CSO volumes with an annual frequency of occurrence.

\subsubsection{Sources of Uncertainty}

The four sources of uncertainty are described below and detailed descriptions of the computational procedures for LTSs can be found in the CSO Technical Guidance Manual (MGS, 2010).

\section{Representativeness of Historical 32 y Precipitation Time Series}

Use of the historical precipitation time series record to model the characteristics of future precipitation produces uncertainties in model-predicted flows for 
future conditions. Assuming a stationary climate, the longer the historical record, the more likely that record will be representative of future storm characteristics. Assessment of the 32 y record using the results from statewide and Seattle-specific precipitation frequency studies results in a standard error of estimation (standard deviation) for precipitation frequency of 5\%. Thus, using conventional sampling statistical theory, the range and distribution of future 32 y precipitation time series can be modeled as the historical 32 y time series rescaled by a precipitation scaling factor with a mean of 1.00 and a standard deviation of 0.05 drawn from a normal distribution.

\section{Possible Effects of Climate Change}

Scientific consensus is that the climate is not stationary but that climate change is underway. The historical 32 y precipitation time series already contains any recent effects of climate change (1978-2009). The primary interest for the future $30 \mathrm{y}$ planning period is what additional changes may occur in the future. A simplified probabilistic model was formulated that considers that precipitation in the future $30 \mathrm{y}$ planning period may range from no change to an increase of $15 \%$, with a most likely value being a $5 \%$ increase. The $5 \%$ increase comes from an estimated $1{ }^{\circ} \mathrm{F}$ change in north pacific sea surface temperature over the next three decades that represents a $1 \stackrel{\circ}{\mathrm{F}}$ change in dew point and a $5 \%$ increase in atmospheric moisture. More information is available from the University of Washington Climate Impacts Group at (http://cses.washington.edu/cig/pnwc/cc.shtml). An empirical likelihood function, developed for use in conducting the uncertainty analysis, is described in the CSO Technical Guidance Manual (MGS, 2010). The range of effects of climate change are mimicked by scaling the historical precipitation time series by a precipitation scaling factor obtained from Monte Carlo selection from the climate change likelihood function.

\section{Model Uncertainties}

Uncertainties exist in model predicted values because of inaccuracies and uncertainties in the model inputs and imperfections in the structure and governing algorithms used to make predictions. The magnitude of uncertainties in predicted sewer flows was estimated using global GOF measures computed during the model calibration process. Ideally, uncertainties in the prediction of sewer flows for the LTSs would be modeled by adjusting the predicted sewer flows at locations within the SWMM5 model. However, this approach is impractical in the SWMM5 model. Alternatively, precipitation scaling factors were used to rescale the historical precipitation time series as a surrogate forcing function to mimic changes in sewer flows. This was a practical approach recognizing the linearity of response of stormwater runoff from impervious areas to small changes in precipitation. 


\section{Residual Uncertainties}

Residual uncertainties account for uncertainties that arise from sources other than the three categories described above. This includes the effect of the number of flow meters, quality of flow data, and representativeness of precipitation used in model calibration. It also includes uncertainties associated with hydraulic components such as HydroBrakes, pump stations, flap gates, overflow weirs, and storage tanks that add to the uncertainty of system performance in the long term future period. The effects of residual uncertainties were mimicked using precipitation scaling factors in the same manner as described above for model uncertainties.

\subsubsection{Amplification Factor, Non-Linearity of Overflow Volumes}

The results from prior uncertainty analyses indicate that the sewer system response for prediction of sewer flows is nearly linear for the magnitude of uncertainties commonly encountered in the Seattle CSS. However, estimates of sewer overflow volumes are often highly nonlinear because sewer overflows are the diverted portion of a larger flow in the main line. Small increases in the magnitude of sewer flow in the inflow main line can produce relatively large increases in the magnitude of diverted flow that overflows. This nonlinearity in the prediction of overflow volumes, termed the amplification factor, is a characteristic of a specific overflow structure configuration and magnitude of inflows. For example, if actual sewer flows are 10\% larger than predicted, the majority of the $10 \%$ excess could be directed to the overflow weir and result in a $50 \%$ increase in the overflow volume. For this case, the amplification factor would be 5.0, a 5:1 ratio relative to uncertainties in sewer flow prediction. The nonlinear behaviour of sewer overflow volumes is the primary cause for wide uncertainty bounds for the best estimate $\mathrm{CSO} \mathrm{CV}$.

\subsubsection{Best Estimates of Control Volumes}

For each basin model, eleven LTSs were conducted using the calibrated basin model and the historical 32 y precipitation time series multiplied by the eleven precipitation scaling factors. These LTSs represent plausible futures based on what might be the conditions in the future for the four sources of uncertainty.

ACU-SWMM computes the volume and duration of each overflow event simulated during the $32 \mathrm{y}$ period for each LTS. The collection of overflows is ranked from largest to smallest and the annual CV for each LTS is computed. The best estimate CSO CV is computed as the mean of the eleven CVs for the eleven LTSs. Uncertainty bounds are computed by fitting a three-parameter Gamma Distribution to the eleven CVs. The results of these analyses are graph- 
ically depicted in Figure 17.6 below. The graph shows that the CV best estimate is $1.858 \mathrm{MG}$ with an amplification factor of 1.38 . The amplification factor is a numerical measure used to characterize the non-linearity seen in the overflow volumes due to uncertainties. The higher the number, the greater the nonlinearity of overflow volumes that result from the four sources of uncertainties.

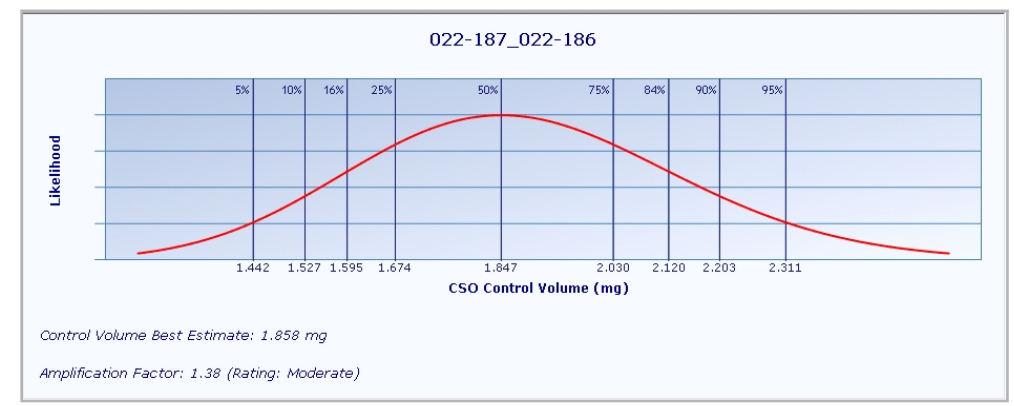

Figure 17.6 Graphic results from LTS prediction of control volume.

\subsection{Case Study on the Interbay CSO Area}

\subsubsection{Overview}

The Interbay CSO Area covers 290 acres (118 ha) in northwest Seattle (see Figure 17.7 below). The Interbay CSO Area comprises the NPDES068(A) and NPDES068(B) subbasins, which drain from east to west and then north toward the King County north trunk line. Each subbasin has a single overflow point with its own NPDES designation, but overflows are reported as a combination of both and listed as NPDES068 in the city's CSO reports. The wastewater generated in these basins flows by gravity to King County's north trunk line for conveyance to the West Point wastewater treatment plant.

The CSS in the Interbay CSO area conveys both sanitary and stormwater flow. Just over three quarters of the Interbay CSO area is partially separated. Storm sewers collect and convey street runoff and a portion of private property runoff. Stormwater from partially separated areas of the Interbay CSO area is discharged into Elliot Bay. The NPDES068(A) and NPDES068(B) subbasins contain permitted CSO structures that discharge overflows into the drainage system that discharges to Elliot Bay during large precipitation events when the capacity of the CSS is exceeded.

The Interbay CSO area was modeled using the SWMM5 platform. The model was initially constructed as one large model and then broken down into the two subbasins for automated calibration and LTS. The automated calibra- 
tion process utilized flow monitoring data from five sites and manual calibration utilized flow monitoring data from an additional three sites.

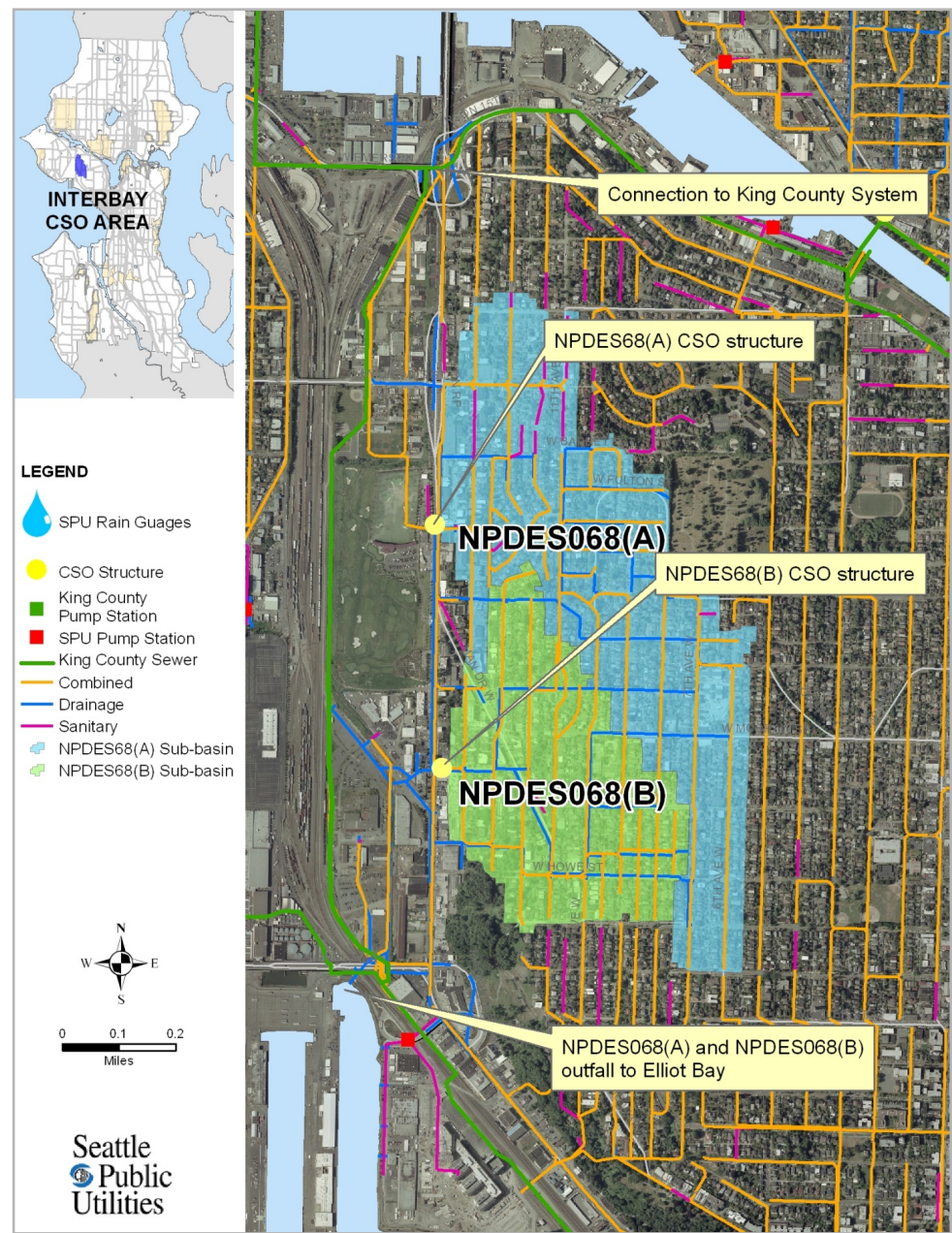

Figure 17.7 Interbay CSO area overview.

\subsubsection{Automated Calibration Results}

Each of the two subbasins was broken into calibration subcatchments. In the NPDES068(A) subbasin, two upper calibration subcatchments were calibrated through ACU-SWMM. In the NPDES068(B) subbasin, one calibration subcatchment upstream was calibrated using ACU-SWMM. Because of limited flow monitoring data during the November 2009 and January 2010 storm 
events in the NPDES068(A) subbasin, the groundwater parameters in both subbasins were calibrated based on a flow monitor in the NPDES068(B) subbasin. Table 17.2 summarizes the best fit parameter sets and the GOF values for the subcatchment calibration groups.

Table 17.2 Best fit parameter sets and goodness of fit values.

\begin{tabular}{|c|c|c|c|c|c|c|c|c|c|c|}
\hline 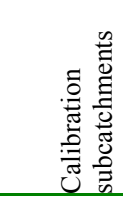 & 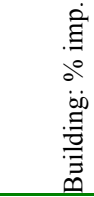 & 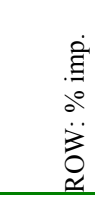 & 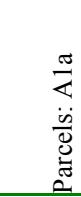 & 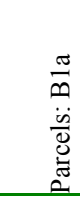 & 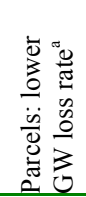 & 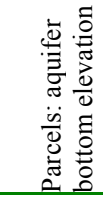 & 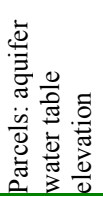 & $\begin{array}{l}\overrightarrow{8} \\
\stackrel{8}{0} \\
0 \\
0 \\
0\end{array}$ & 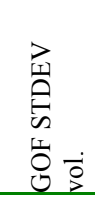 & 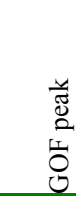 \\
\hline $021-364$ & 14.625 & 31.125 & 0.001 & 1.019 & 0.683 & 66.448 & 66.897 & 0.850 & 0.018 & 1.248 \\
\hline $3-517 b$ & 33.160 & 70.060 & 1 & 1.0 & 33 & 125.048 & & 17 & 0.095 & 1.051 \\
\hline $028-043 \mathrm{c}$ & -- & -- & & 1.01 & 3 & & & 97 & 0.072 & 1.147 \\
\hline $028-430$ & 43.670 & 40.580 & 0.001 & 1.019 & 0.683 & 27.498 & 27.947 & 1.000 & 0.072 & 1.015 \\
\hline
\end{tabular}

a. Groundwater parameters for the entire Interbay CSO Area were determined from flow data collected at $\mathrm{MH}$ 028-043 because the meter was in place for a longer duration and collected data during storms that showed groundwater signatures.

b. Best fit parameters for impervious area were modified in 0.28-517 during manual calibration. This table lists the values for impervious area that were used for the LTS.

c. Model 028-043 was used for groundwater parameter calibration only. The impervious values were locked in based on the results from $028-430$.

\subsubsection{Long term Simulation Results}

For the LTS, the Interbay CSO area model was broken down into three models to facilitate the use of larger time steps for the hydrologic upper basin in the NPDES068(A) subbasin. Figure 17.8 shows the breakdown of the Interbay CSO Area model for LTS.

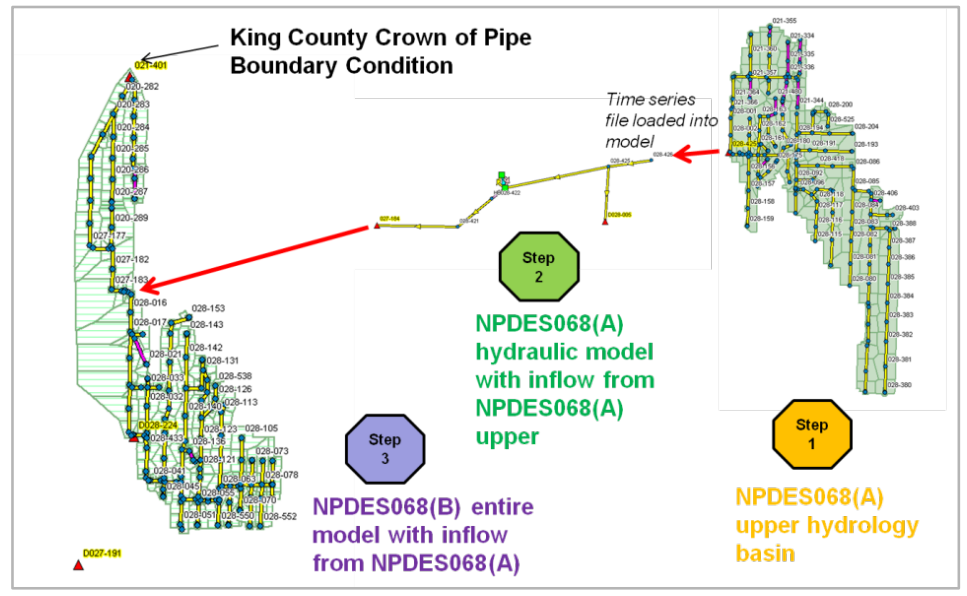

Figure 17.8 Interbay CSO area models for LTS. 
The larger time step could not be used for the hydraulic structures in the lower portion of the basin due to model instabilities. The upper basin model could run twice as fast using the larger time step. In addition, the model was extended to the King County system downstream of the overflow in NPDES068(B) to set a boundary condition. No boundary condition was needed for the NPDES068(A) model as a drop structure downstream of the overflow structure prevented any downstream influence on the model.

Table 17.3 summarizes the residual uncertainties developed for Interbay based on the four sources of uncertainty described in Section 17.3.1. These values were used to develop the eleven scaling factors for the precipitation time series.

Table 17.3 Summary of residual uncertainties from all sources.

\begin{tabular}{lll}
\hline \multirow{2}{*}{ Uncertainty statistic } & \multicolumn{2}{l}{ Residual uncertainty statistics for uncertainty analyses } \\
\cline { 2 - 3 } & NPDES068(A) & NPDES068(B) \\
\hline Mean value of sewer flows & 1.000 & 1.000 \\
Standard deviation of sewer flows & 0.114 & 0.064 \\
\hline
\end{tabular}

Table 17.4 provides the derived eleven precipitation scaling factors developed from the residual uncertainties and the resulting corresponding once-peryear volumes, in million gallons, simulated for the NPDES068(A) Subbasin. Figure 17.9 provides a graphic display of the CV uncertainty statistics, best estimate $\mathrm{CV}$, and corresponding amplification factor. For the NPDES068(A) subbasin, the best estimate $\mathrm{CV}$ was calculated at $0.056 \mathrm{MG}$, with an amplification factor of 9.31. Table 17.5 provides the derived eleven precipitation scaling factors and the resulting corresponding once-per-year volumes, in million gallons, simulated for the NPDES068(B) subbasin.

Table 17.4 NPDES068(A) subbasin long term results.

\begin{tabular}{ll}
\hline Precipitation scale factor (suggest 3 decimal places) & Once per year volume (MG) \\
\hline 0.79 & 0 \\
0.8773 & 0 \\
0.934 & 0.0009 \\
0.9855 & 0.0048 \\
1.0106 & 0.0069 \\
1.051 & 0.0233 \\
1.0947 & 0.0456 \\
1.1352 & 0.0687 \\
1.1717 & 0.0949 \\
1.2475 & 0.1483 \\
1.3487 & 0.2261 \\
\hline
\end{tabular}




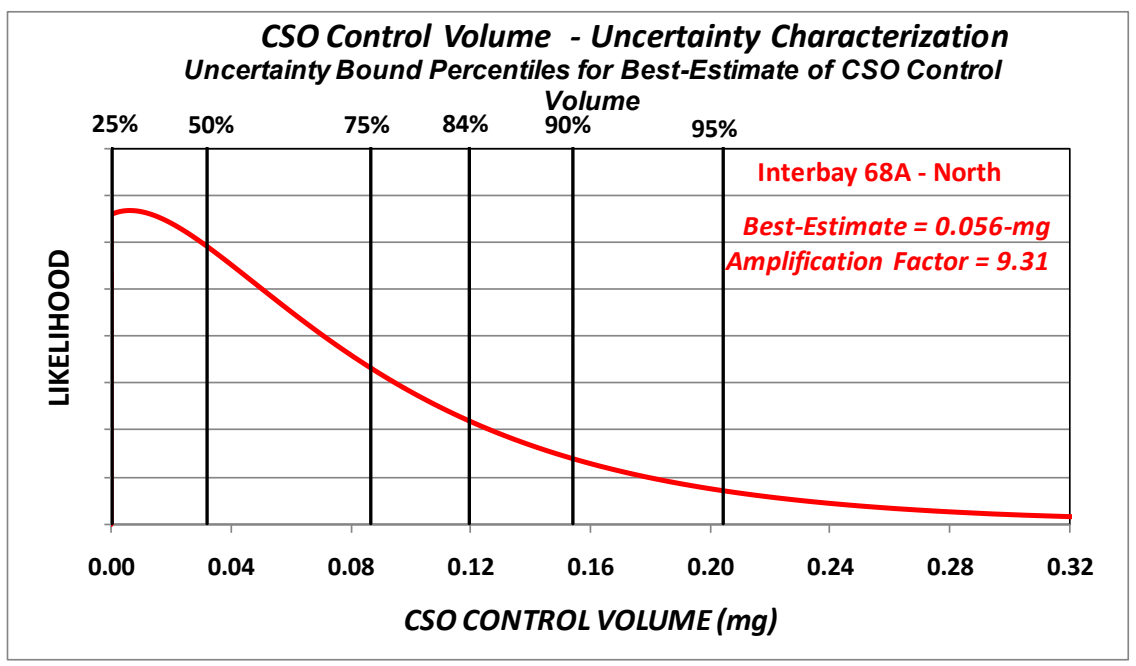

Figure 17.9 NPDES068(A) control volume best estimate, uncertainty statistics and amplification factor.

Table 17.5 NPDES068(B) sub basin long term results.

\begin{tabular}{ll}
\hline Precipitation scale factor (3 decimal places) & Once per year volume (MG) \\
\hline 0.9018 & 0 \\
0.9657 & 0 \\
0.9957 & 0 \\
1.0138 & 0 \\
1.0342 & 0 \\
1.0583 & 0 \\
1.0802 & 0 \\
1.1 & 0 \\
1.1275 & 0 \\
1.1595 & 0.0018 \\
1.2237 & 0.0179 \\
\hline
\end{tabular}

The uncertainty analysis indicates that the NPDES site would be controlled for nine of the eleven plausible futures. CVs were generated for only two of the eleven plausible futures. Therefore, it is very likely that the NPDES site would meet the required annual overflow standard with a small chance that the site is actually uncontrolled. In the two cases where CVs were generated, the overflow volumes were highly nonlinear with regard to the sewer flows in the system. The amplification factor has been judged to be extreme and assigned a nominal value of greater than 20 . Figure 17.10 provides a graphic display of the CV uncertainty statistics, best estimate $\mathrm{CV}$, and corresponding amplification factor. For the NPDES068(B) subbasin, the best estimate CV was calculated at 0.002 $\mathrm{MG}$, with an amplification factor greater than 20 . 


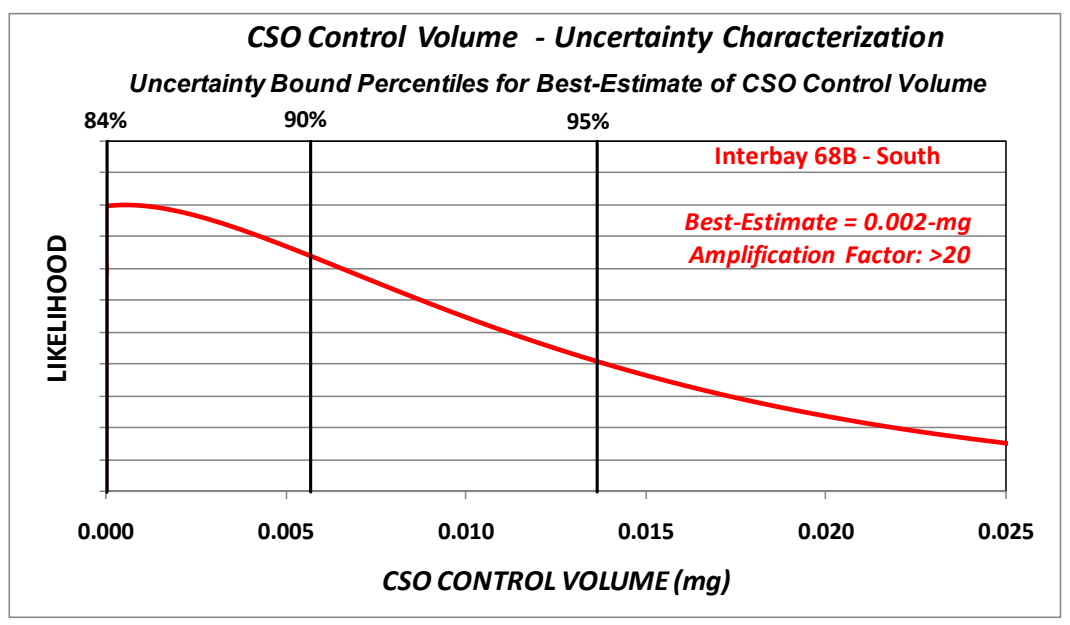

Figure 17.10 NPDES068(B) control volume best estimate, uncertainty statistics, and amplification factor.

It should be noted that in the majority of NPDES basins, the uncertainty bounds for $\mathrm{CV}$ were similar to those seen in Figure 17.6. The Interbay basins were somewhat unusual in that the long term simulations indicated a high likelihood of compliance and the uncertainty bounds for CV represented only those plausible futures where a $\mathrm{CV}$ was required.

\subsection{Conclusions}

The CSO CV is the volume of overflow that is exceeded an average of once per year in the 32 years of model simulation. The City will evaluate alternatives such as GSI and retrofits to reduce the overall volume of water the city will need to store.

Analysis of the operation of CSSs and estimating the CV for CSO reduction are difficult technical challenges. The difficulty in estimating the CV stems from two primary reasons:

1. Storms in any given period over the past 60 years in the Seattle area have shown very high variability with regard to their frequency, magnitude, and temporal characteristics; and

2. Sizable uncertainties are associated with flow monitoring data, various analyses, and computer modeling of CSSs. These uncertainties generally have a non-linear effect that acts to amplify uncertainties in the estimation of overflow volumes and widen the uncertainty bounds for the CSO CV. 
The city cannot change the rainfall or regulations associated with reason 1 above. However, Seattle succeeded at reducing the sizable uncertainties associated with reason 2 through a rigorous flow monitoring program, the use of automated calibration, and performing uncertainty analysis prior to long term hydraulic and hydrologic model simulations. In addition, the city quantified the uncertainties to better understand the potential risk and cost associated with $\mathrm{CV}$ estimates due to these uncertainties. For instance, if the city decided to control CSOs with $90 \%$ confidence, storage for $2.203 \mathrm{MG}$ would be needed, using the results shown in Figure 17.6. This would lessen the risk that the city would violate its NPDES permit for a larger cost than controlling the best estimate of $1.858 \mathrm{MG}$. This level of detail in developing CV estimates provides the city with a suitable tool for the evaluation of cost and risk associated with control of CSOs. The city is working on a long term control plan that will define the CSO reduction projects that will happen from 2016 to 2025.

\section{References}

Beven KJ and Binley A, 1992. The Future of Distributed Models: Model Calibration and Uncertainty Prediction, Hydrological Processes, Volume 6, pp 272-298, 1992.

MGS Engineering Consultants, Inc. 2010. CSO Technical Guidance Manual, Chapters 5 and 6, prepared for Seattle Public Utilities by MGS Engineering Consultants Inc., March 2010.

\section{Background Sources}

CH2M HILL, Brown and Caldwell, GHD, 2010d. SPU Long term Control Plan Flow Monitoring Report Volume 5: Phases 2-3 Flow Monitoring Report.

CH2M HILL, Brown and Caldwell, GHD, 2010e. Draft Uncontrolled CSO Basin Modeling Plan Long term Control Plan Basin Model Development.

MGS Engineering Consultants, Inc. 2003. Analysis of Precipitation-Frequency and Storm Characteristics for the City of Seattle. Prepared for Seattle Public Utilities. December 2003.

MGS Engineering Consultants, Inc. 2009. Methods for Estimating Control Volumes for CSO Reduction: Technical Guidance Manual. Prepared for Seattle Public Utilities. February 2009.

USEPA, 2010. Storm Water Management Manual User's Manual. Version 5.0. EPA/600/R-05/040. Revised July 2010. 